\title{
Optical Coherence Tomography Prognostic Factors in Age-Related Macular Degeneration Patients with Peripapillary Choroidal Neovascularization
}

\author{
Gilad Rabina $^{a}$ Anfisa Ayalon $^{b}$ Michael Mimouni $^{c}$ Nir Stanescu ${ }^{b}$ \\ Elad Moisseiev ${ }^{b}$ Arie Y. Nemet ${ }^{b}$ Noa Geffen ${ }^{d}$ Ori Segal $^{b}$ \\ aDepartment of Ophthalmology, Tel Aviv Sourasky Medical Center, Affiliated to the Sackler Faculty of Medicine, \\ Tel Aviv University, Tel Aviv, Israel; ' bepartment of Ophthalmology, Meir Medical Center, Affiliated to the Sackler \\ Faculty of Medicine, Tel Aviv University, Kfar Saba, Israel; 'Department of Ophthalmology, Rambam Health Care \\ Campus, Haifa, Israel; ${ }^{d}$ Department of Ophthalmology, Rabin Medical Center, Petah Tikva, Israel
}

\author{
Keywords \\ Choroidal neovascularization · Peripapillary · Age-related \\ macular degeneration - Optical coherence tomography . \\ Prognostic signs
}

\begin{abstract}
Purpose: The aim of the study was to investigate the correlation between optical coherence tomography (OCT) findings and visual acuity outcomes after treatment with intravitreal bevacizumab (IVB) injections for age-related macular degeneration (AMD) patients with peripapillary choroidal neovascularization (PPCNV). Methods: The study involved a retrospective case series of consecutive patients diagnosed with PPCNV secondary to AMD. All patients were treated with IVB injections with a follow-up time of 1 year. Data collected included best-corrected visual acuity (BCVA) and automated and manually measured OCT parameters. Results: A total of 68 eyes were diagnosed with PPMV. Of them, 30 eyes of 30 patients aged $84.3 \pm 6.9$ years of which $63.3 \%$ female gender were included. Baseline BCVA was $0.46 \pm 0.62 \log M A R$ (Snellen 20/57), average choroidal thickness was $193.2 \pm 22 \mu \mathrm{m}$, and mean number of IVB injections was 7.2 \pm 1.9 . After 1 year, BCVA was $0.56 \pm 0.78 \log M A R$ (Snellen $20 / 72)(p=0.28)$. Eyes
\end{abstract}

Karger@karger.com www.karger.com/oph

Karger $\stackrel{\text { ' }}{5}$

BOPEN ACCESS
(C) 2021 The Author(s)

Published by S. Karger AG, Basel

This is an Open Access article licensed under the Creative Commons Attribution-NonCommercial-4.0 International License (CC BY-NC) (http://www.karger.com/Services/OpenAccessLicense), applicable to the online version of the article only. Usage and distribution for commercial purposes requires written permission. with greater central retinal thickness $(r=-0.36, p=0.05)$, greater subretinal hyper-reflective material (SHRM) area $(r=$ $-0.37, p=0.05)$, and greater sub-retinal fluid (SRF) area ( $r=$ $-0.73, p<0.001$ ) had a significantly smaller improvement in BCVA. Eyes with pigment epithelium detachment (PED) $(0.68$ \pm 0.90 vs. $0.21 \pm 0.12, p=0.03$ ) had a significantly worse BCVA. Conclusions: Our data suggest that AMD-related PPCNV with greater foveal thickness, PED size, SHRM, and SRF areas have worse final BCVA prognosis.

(C) 2021 The Author(s) Published by S. Karger AG, Basel

\section{Introduction}

Peripapillary choroidal neovascularization (PPCNV) is a rare condition with a population prevalence of $0.29 \%$ [1]. It is defined as a choroidal neovascularization (CNV) found within 1-disc diameter or less from the optic nerve disc [2], and it accounts for $10 \%$ of all $\mathrm{CNV}$ with a predisposition toward females [3]. PPCNV can be symptomatic with disturbance in visual acuity or asymptomatic and discovered by routine follow-up, usually depending on $\mathrm{CNV}$ location.

Gilad Rabina and Anfisa Ayalon share equal contribution.
Correspondence to:

Gilad Rabina, giladrabina@ hotmail.com 
There are various conditions that have been shown to be associated with PPCNV, such as age-related macular degeneration (AMD), polypoidal choroidal vasculopathy (PCV), myopia, angioid streaks, birdshot chorioretinopathy, sarcoidosis, serpiginous choroiditis, optic disc malformations, optic disc drusen, and causes [4]. Treatment in the past included laser [5] and vitrectomy with $\mathrm{CNV}$ extraction for large lesions [6]. Later on, photodynamic therapy with verteporfin [7] was the mainstay treatment, and in recent years anti-vascular endothelial growth factor (VEGF) drugs play an important role in PPCNV treatment [8]. There is a paucity of data on optical coherence tomography (OCT) prognostic signs for patients with PPCNV treated with anti-VEGF. The purpose of this study was to investigate the correlation between spectral-domain OCT signs at baseline and visual acuity outcomes after treatment with intravitreal bevacizumab (IVB) injections for neovascular AMD patients with PPCNV.

\section{Methods}

The study adhered to the tenets of the Declaration of Helsinki and was approved by the Institutional Review Board (IRB) of the Meir Medical Center.

\section{Study Participants}

This retrospective study included consecutive patients that were diagnosed with PPCNV related to AMD between January 2012 and July 2019 at Meir Medical Center, Kfar Saba, Israel. All patients were treatment-naive at diagnosis and treated with 3 consecutive injections followed by pro re nata treatment and with a follow-up time of at least 12 months.

\section{Data Collection}

Cases were identified by electronic medical records and patients with PPCNV temporal to the optic disc and secondary to AMD were included. The diagnosis of AMD was made by clinical examination, SD-OCT (Spectralis OCT; Heidelberg Engineering, Heidelberg, Germany), fluorescein angiography (FA) (Heidelberg Engineering, Heidelberg, Germany and/or Optos plc, Dunfermline, UK), and indocyanine green angiography (ICGA) (Heidelberg Engineering, Heidelberg, Germany and/or Optos plc, Dunfermline, UK) to rule out other possible pathologies such as PCV. A $\mathrm{CNV}$ was defined as the presence of pigment epithelial detachment (PED) and presence of intraretinal fluid (IRF), subretinal fluid (SRF), and subretinal hyper-reflective material (SHRM) OCT [912], hemorrhage and drusen on clinical examination, or leakage on FA consistent with neovascularization [13]. Patients with macular involvement or with any CNV findings on the OCT exam with visual symptoms including decrease in visual acuity or metamorphopsia were treated with anti-VEGF injections. Patients with PPCNV nasal to the optic disc were excluded from this study and were treated according to retina specialist discretion. Re-treatment criteria included recurrence of IRF, SRF, and SHRM on OCT or hemorrhage on clinical examination.
The medical files of all patients were reviewed and the following information extracted: age, gender, best-corrected visual acuity (BCVA), involved eye, and slit-lamp examination including CNV location relative to the optic nerve disc, determined by OCT and FA or ICGA. BCVA was assessed at baseline and at each clinic visit. Final BCVA was assessed 1 month after the last bevacizumab injection.

For AMD patients, in general, choroidal thickness is usually below $200 \mu \mathrm{m}$ and thicker for the spectrum of pachychoroid diseases such as PCV or CSR [14]. In order to exclude PCV and CSR, patients with choroidal thickness of $<250 \mu \mathrm{m}$ were excluded from this study. Eyes with high myopia of above $6 \mathrm{D}$, history of retinal detachment, macular telangiectasias, tractional and degenerative lamellar macular holes, central or branch retinal vein occlusion, central or branch retinal artery occlusion, advanced glaucoma, or optic neuropathy of any kind, visually significant cataract, endophthalmitis, retinal dystrophies, inability to perform OCT or any cause for nonreadable OCT, and any previous intraocular surgery besides uncomplicated cataract surgery were also excluded from this study.

\section{OCT Processing}

The SD-OCT testing was performed through a dilated pupil, centered on the fovea, using the established "posterior pole" protocol that provides high-speed scans with a dimension of $30^{\circ} \times 25^{\circ}$ and a $120-\mathrm{mm}$ B-scan spacing and "optic disc" protocol with scans centered on the optic disc with a dimension of $15^{\circ} \times 15^{\circ}$ and a 120 $\mathrm{mm}$ B-scan spacing. All OCT images were carefully reviewed by 2 out of 3 masked retina specialists. If there was disagreement of $<10 \%$ between the 2 graders, the average value was used, and if the disagreement was $>10 \%$, then adjudication by a third grader would take place. The maximum width of each of the following was measured using the caliper scale provided by the software of the SD-OCT device: SRF, IRF, PED, and SHRM [15]. In addition, the maximum height and/or area of the following were marked and measured using the built-in caliper scale and overlay function (enables to display and measure the area marked): SRF, IRF, PED, SHRM, subfoveal choroidal thickness, and CNV lesion. The vitreous status including complete attachment, vitreomacular adhesion (VMA), or partial posterior vitreous detachment (PVD) was documented. In addition, the following parameters were recorded from the automatic calculation of the SD-OCT after segmentation error corrections: average central retinal thickness (CRT), maximum CRT (max CRT), and minimum CRT (min CRT) in the central $6 \mathrm{~mm}$. The follow-up mode of the eye tracking-assisted system (AutoRescan) was used for follow-up visits. As previously described, the PED was defined as an inward deviation of the normal counter of the retinal pigment epithelium (RPE) with an optically empty area externally and was measured from the top of the RPE to the top of the Bruch's membrane [16]. The SRF was defined as the presence of an optically empty area directly internal to the RPE/Bruch membrane complex and external to the outer retina, whereas IRF was defined as the presence of an optically empty area within the retina. The presence of SHRM was defined as the presence of hyper-reflective material located external to the retina and internal to the RPE. The dimensions of CNV lesion were defined as presence of hyper-reflective lesion internal to the Bruch membrane and external to the retina with SRF and/or IRF and/or PED adjacent to the edges of hyper-reflective area. The presence of hyper-reflective foci (HF) was defined as the presence 

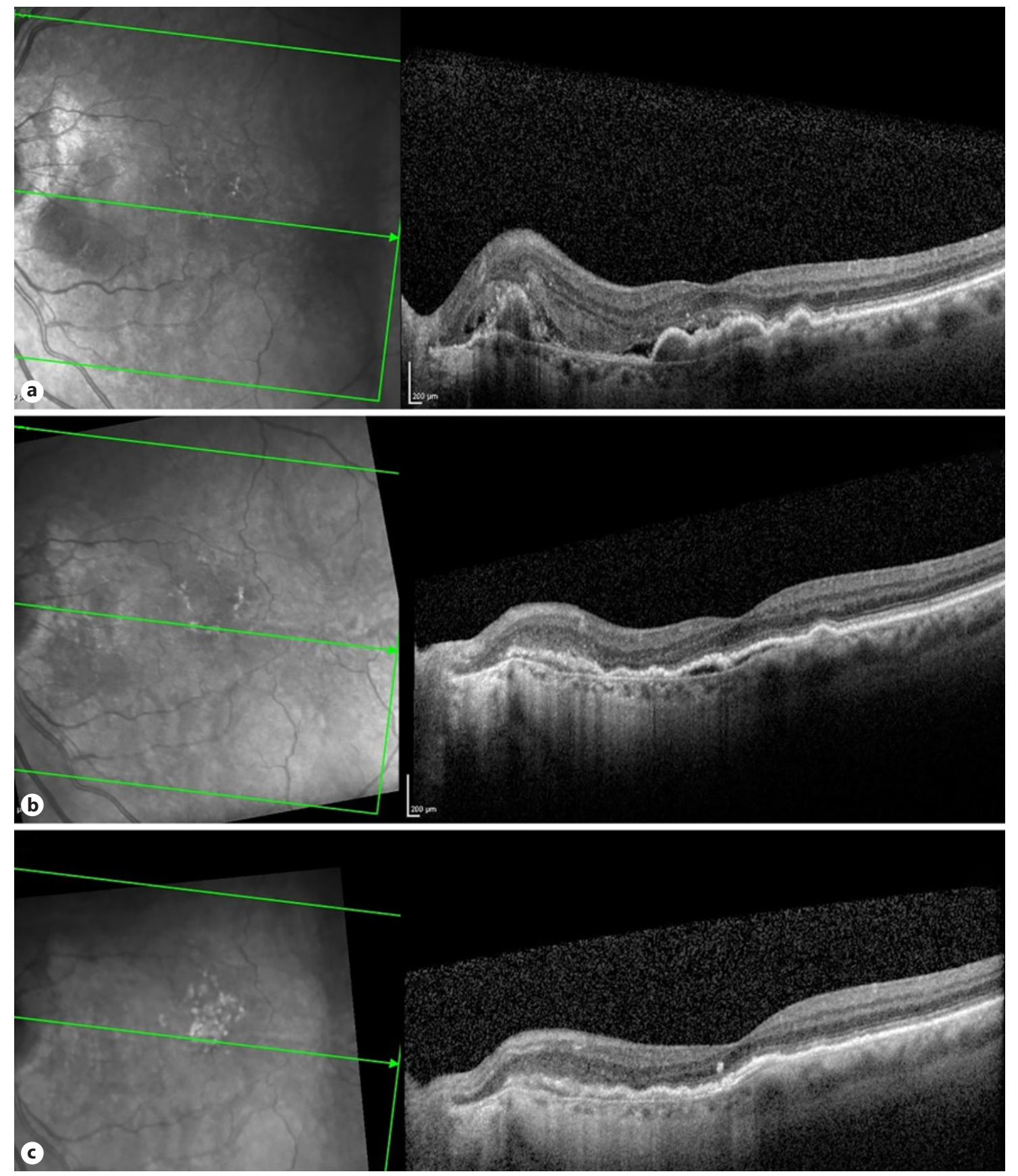

Fig. 1. Patient No. 15. Ninety-four-year-old female with left-eye PPCNV secondary to AMD, with foveal involvement. The BCVA at presentation was Snellen 20/40 and improved to Snellen 20/25 during the 12 months of follow-up. The patient underwent a total of 7 bevacizumab injections. a At Presentation. b After 3 months of follow-up. c After 12 months of follow-up.

of small focal hyper-reflective material (as hyper-reflective as RPE) observed in at least one available scan. The B-scan with most HF was evaluated to determine the amount of HF. The amount of HF was classified as few (0-10), moderate (11-20), or many $(>20)$ as previously described [10].

\section{Statistical Analysis}

All data collected in the study were inserted into an electronic database via Microsoft Excel 2013 (Microsoft Corporation). Statistical analyses were performed using Minitab Software, version 17 (Minitab Inc, State College, PA, USA). Results are expressed as 
Fig. 2. Patient No. 15. FA (a, b) and ICGA (c, d). a $30 \mathrm{~s}$, after venous stage. b 8:00 min, late stage. A hyper-fluorescent plaque is demonstrated temporal to the optic disc, corresponding to CNV. Areas of hyper fluorescence corresponding to the PED's noticed on the OCT. c 1:00 min, early phase. d 10:00 min, middle phase. Peripapillary areas of leakage, corresponding to $\mathrm{CNV}$ with late macular leakage. No evidence of polyps.
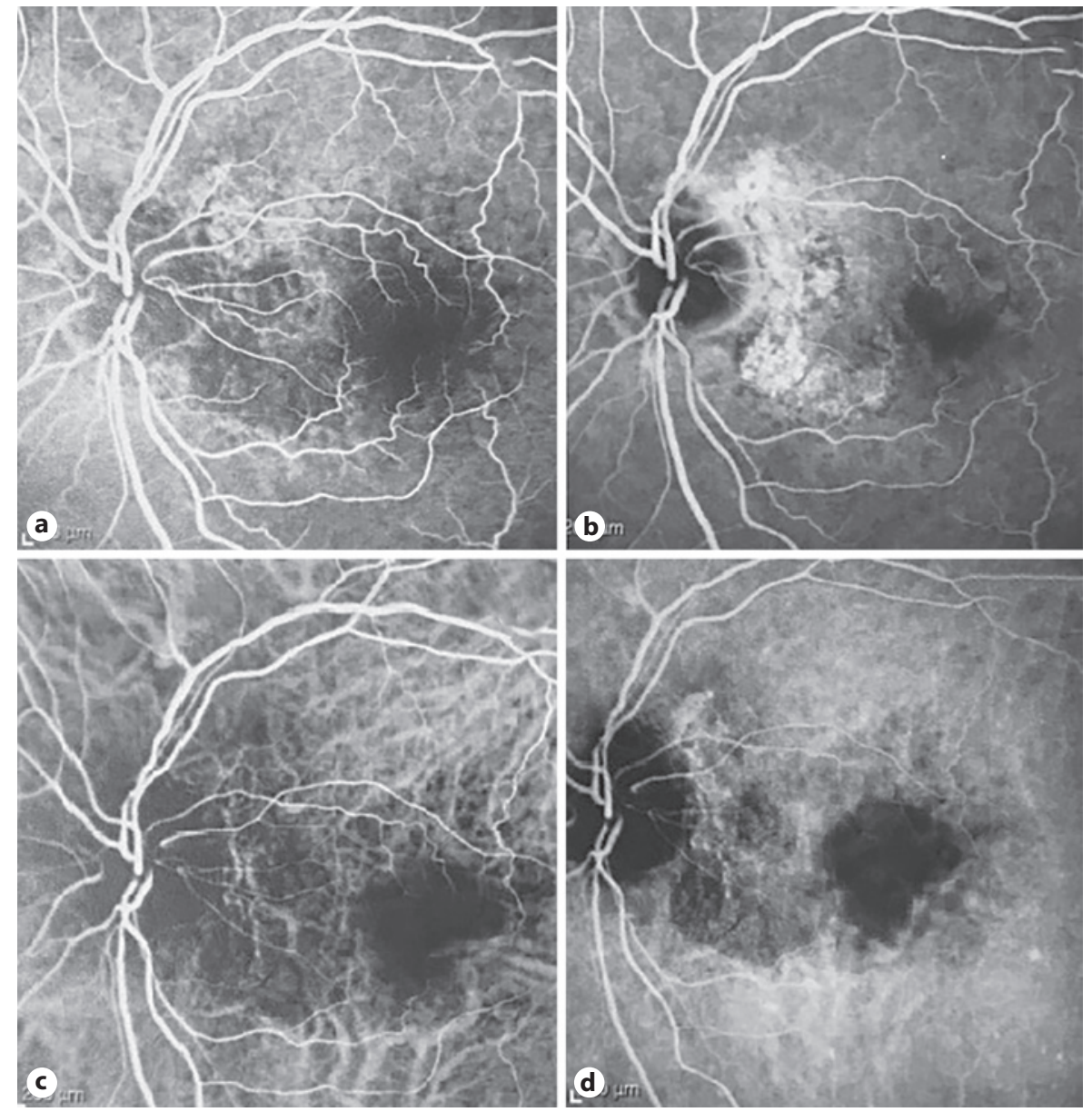

mean $\pm \mathrm{SD}$, median (range) or $N(\%)$. Pearson correlation was used to analyze the relationship between baseline parameters and delta BCVA as well as 1-year BCVA. Baseline OCT characteristics were compared by using, whenever appropriate, Student $t$ test for normally distributed variables or Kruskal-Wallis for nonparametric variables. $\chi^{2}$ or Fishers' exact test were used as indicated for analysis of categorical variables. One-way analysis of variance was used for comparison of multiple group averages. Multiple regression analysis was performed to determine the baseline variables that enabled to predict 1-year BCVA. For this purpose, we introduced as independent variables those variables that reached a significant level of $<0.05$ in univariate analysis. A $p$ value of $<0.05$ was considered statistically significant.

\section{Results}

A total of 68 eyes were diagnosed with PPMV during the study period. Of them, 38 were excluded due to CNV location nasal to the optic disc, choroidal thickness above $250 \mu \mathrm{m}, \mathrm{CNV}$ secondary to other etiology then AMD, insufficient follow-up time and insufficient quality of OCT images. Overall, 30 eyes of 30 patients aged $84.3 \pm 6.9$ years (range 70-98 years) of which $63.3 \%$ were of female gender were included in this study. The CNV was located in the superior half in $73.3 \%$ of cases and the baseline logMAR BCVA was $0.46 \pm 0.62$ (Snellen 20/57). Foveal involvement was noted in 15 (50\%) eyes, average choroidal thickness was $193.2 \pm 22 \mu \mathrm{m}$, and mean number of intravitreal injections was $7.2 \pm 1.9$ (range $3-10$ ) with $70 \%$ of patients requiring further injections after 1 year of treatment. Figure 1 depicts OCT images of patient No. 15, with PPCNV with foveal involvement. Figure 2 depicts FA and ICGA images of patient No. 15, PCV was ruled out.

After 1 year of treatment, there was no significant change in BCVA $(0.46 \pm 0.62$ vs. $0.56 \pm 0.78, p=0.28)$ (Snellen $20 / 57$ vs. 20/72). Table 1 depicts correlations between categorical baseline parameters and change in BCVA as well as final BCVA at 1 year. Briefly, there was no significant correlation between categorical baseline features and change in BCVA at 1 year. Eyes 
Table 1. Correlations between categorical baseline parameters and change in BCVA from baseline (delta) as well as logMAR BCVA at 1 year

\begin{tabular}{|c|c|c|c|c|}
\hline \multirow[t]{2}{*}{ Parameter } & \multicolumn{2}{|c|}{ Delta logMAR BCVA } & \multicolumn{2}{|c|}{ 1-year logMAR BCVA } \\
\hline & mean $\pm S D$ & $p$ value $^{a}$ & mean $\pm S D$ & $p$ value $^{a}$ \\
\hline \multicolumn{5}{|l|}{ Gender } \\
\hline Male & $-0.02 \pm 0.17$ & \multirow{2}{*}{0.40} & $0.28 \pm 0.21$ & \multirow{2}{*}{0.06} \\
\hline Female & $-0.15 \pm 0.63$ & & $0.73 \pm 0.94$ & \\
\hline \multicolumn{5}{|l|}{ ERM } \\
\hline Yes & $-0.14 \pm 0.73$ & \multirow{2}{*}{0.78} & $0.66 \pm 0.86$ & \multirow{2}{*}{0.57} \\
\hline No & $-0.08 \pm 0.27$ & & $0.49 \pm 0.73$ & \\
\hline \multicolumn{5}{|l|}{ CNV location } \\
\hline Superior half of macula & $-0.14 \pm 0.59$ & \multirow{2}{*}{0.25} & $0.68 \pm 0.89$ & \multirow{2}{*}{0.04} \\
\hline Inferior half of macula & $0.01 \pm 0.13$ & & $0.25 \pm 0.16$ & \\
\hline \multicolumn{5}{|c|}{ Peripapillary vitreous adherence } \\
\hline Yes & $0.04 \pm 0.33$ & \multirow{3}{*}{0.43} & $0.43 \pm 0.53$ & \multirow{3}{*}{0.47} \\
\hline No & 0.10 & & $0.64 \pm 0.95$ & \\
\hline Not seen & $-0.21 \pm 0.62$ & & & \\
\hline \multicolumn{5}{|l|}{ Vitreous macular adherence } \\
\hline Yes & $-0.04 \pm 0.17$ & \multirow{3}{*}{0.21} & $0.29 \pm 0.22$ & \multirow{3}{*}{0.48} \\
\hline No & $0.37 \pm 0.55$ & & $0.83 \pm 1.02$ & \\
\hline Not seen & $-0.20 \pm 0.60$ & & $0.61 \pm 0.92$ & \\
\hline \multicolumn{5}{|l|}{ SHRM } \\
\hline Yes & $-0.18 \pm 0.55$ & \multirow{2}{*}{0.12} & $0.59 \pm 0.87$ & \multirow{2}{*}{0.70} \\
\hline No & $0.09 \pm 0.36$ & & $0.49 \pm 0.58$ & \\
\hline \multicolumn{5}{|l|}{ PED } \\
\hline Yes & $-0.14 \pm 0.62$ & \multirow{2}{*}{0.34} & $0.68 \pm 0.90$ & \multirow{2}{*}{0.03} \\
\hline No & $0.00 \pm 0.10$ & & $0.21 \pm 0.12$ & \\
\hline \multicolumn{5}{|l|}{ SRF } \\
\hline Yes & $-0.11 \pm 0.59$ & \multirow{2}{*}{0.54} & $0.63 \pm 0.88$ & \multirow{2}{*}{0.06} \\
\hline No & $-0.03 \pm 0.11$ & & $0.24 \pm 0.13$ & \\
\hline IRF & & & & \\
\hline Yes & $-0.40 \pm 0.80$ & & $0.95 \pm 1.20$ & \\
\hline No & $0.05 \pm 0.25$ & 0.14 & $0.35 \pm 0.41$ & 0.18 \\
\hline Hyper-reflective foci $>10$ & & & & \\
\hline Yes & $-0.13 \pm 0.57$ & & $0.44 \pm 0.70$ & \\
\hline No & $-0.03 \pm 0.45$ & 0.60 & $0.67 \pm 0.90$ & 0.47 \\
\hline Macular peripapillary SRF & & & & \\
\hline Yes & $-0.09 \pm 0.55$ & & $0.55 \pm 0.84$ & \\
\hline No & $-0.10 \pm 0.22$ & 0.95 & $0.43 \pm 0.22$ & 0.54 \\
\hline Macular peripapillary IRF & & & & \\
\hline Yes & $-0.31 \pm 0.70$ & & $0.80 \pm 1.06$ & \\
\hline No & $0.06 \pm 0.27$ & 0.11 & $0.35 \pm 0.44$ & 0.19 \\
\hline
\end{tabular}

a Student $t$ test or ANOVA used wherever appropriate. with CNV in the superior half $(0.68 \pm 0.89$ vs. $0.25 \pm$ $0.16, p=0.04)$ and eyes with a PED (0.68 \pm 0.90 vs. 0.21 $\pm 0.12, p=0.03)$ had a significantly worse final logMAR BCVA.

Table 2 depicts correlations between continuous baseline parameters and change in BCVA as well as final BCVA at 1 year. Briefly, eyes with greater CRT $(r=-0.36$, $p=0.05)$, min CRT $(r=-0.45, p=0.01)$, and max CRT $(r=-0.39, p=0.04)$ had a significantly smaller improvement in $\log$ MAR BCVA at 1 year. Similarly, eyes with a greater SHRM area $(r=-0.37, p=0.05)$ and SRF area $(r=-0.73, p<0.001)$ had a significantly smaller improvement in BCVA at 1 year. Eyes with worse vision $(r=0.76$, $p<0.001)$, CRT $(r=0.88, p<0.001)$, $\min$ CRT $(r=0.88$, $p<0.001)$, max CRT $(r=0.89, p<0.001)$, SHRM area $(r=0.55, p=0.002)$, CNV lesion area $(r=0.76, p<0.001)$, PED area $(r=0.56, p=0.002)$, PED height $(r=0.50, p=$ $0.006)$, and SRF area $(r=0.55, p=0.002)$ at baseline had worse 1-year logMAR BCVA.

Table 3 depicts the results of the stepwise multiple regression analysis of factors predicting 1-year BCVA. The factors that remained significant were baseline logMAR 
Table 2. Correlations between continuous baseline parameters and change in BCVA as well as final BCVA at 1 year

\begin{tabular}{|c|c|c|c|c|}
\hline \multirow[t]{2}{*}{ Parameter } & \multicolumn{2}{|c|}{$\begin{array}{l}\text { Delta logMAR } \\
\text { BCVA }\end{array}$} & \multicolumn{2}{|c|}{$\begin{array}{l}\text { 1-year logMAR } \\
\text { BCVA }\end{array}$} \\
\hline & $r$ & $p$ value ${ }^{a}$ & $r$ & $p$ value $^{a}$ \\
\hline Baseline logMAR BCVA & 0.05 & 0.79 & 0.76 & $<0.001$ \\
\hline CRT & -0.36 & 0.05 & 0.88 & $<0.001$ \\
\hline Min CRT & -0.45 & 0.01 & 0.88 & $<0.001$ \\
\hline Max CRT & -0.39 & 0.04 & 0.89 & $<0.001$ \\
\hline SHRM area & -0.37 & 0.05 & 0.55 & 0.002 \\
\hline Fibrovascular complex area & -0.31 & 0.11 & 0.76 & $<0.001$ \\
\hline PED area & -0.17 & 0.39 & 0.56 & 0.002 \\
\hline PED high & -0.36 & 0.06 & 0.50 & 0.006 \\
\hline SRF area & -0.73 & $<0.001$ & 0.55 & 0.002 \\
\hline IRF area & -0.09 & 0.63 & 0.31 & 0.49 \\
\hline
\end{tabular}

a Pearson's correlation.

BCVA $(p=0.05)$, max CRT $(p=0.002)$, SHRM area $(p=$ $0.007)$, and SRF area $(p=0.004)$. The regression equation provided below Table 3 led to an R2 of $89.33 \%$.

\section{Discussion}

In this study, we investigated the correlation between OCT findings at baseline and visual acuity outcomes after treatment with IVB injections in PPCNV secondary to AMD. Although correlation between OCT findings and visual acuity have been investigated in AMD patients with sub-foveal CNV, including the presence of SRF, IRF, SHRM, hyper-reflective foci, outer retinal tubulation, complete RPE and outer retinal atrophy, and PED formation and size $[10,17,18]$, it was, to the best of our knowledge, never reported in the presence of PPCNV.

In this study, after 12 months follow-up and an average of $7.2 \pm 1.9$ anti-VEGF injections there was no significant change in BCVA $(0.46 \pm 0.62$ vs. $0.56 \pm 0.78, p$ $=0.28$ ). Singh et al. [19] recently published 1 year outcomes of patients with PPCNV secondary to AMD, inflammatory, angioid streaks, and idiopathic. They found a statistically significant improvement in BCVA from $0.55 \pm 0.54$ to $0.29 \pm 0.39$ for the entire cohort and $0.43 \pm 0.29-0.32 \pm 0.32$ for AMD patients with an average of $4.9 \pm 2.9$ anti-VEGF injections, but AMD patients needed more injections with an average of $6.1 \pm$ 2.8 [19]. It is worth mentioning that average choroidal thickness in this study was $193.2 \pm 22 \mu \mathrm{m}$ and patients with choroidal thickness of above $250 \mu \mathrm{m}$ were exclud-
Table 3. Multiple regression analysis of baseline parameters predicting 1-year BCVA

\begin{tabular}{llll}
\hline Parameter & $\begin{array}{l}R^{2} \text { (total }= \\
89.33 \%), \%\end{array}$ & Coefficient & $p$ value $^{\mathrm{a}}$ \\
\hline Baseline logMAR BCVA & 56.11 & 0.327 & \\
CRT & 23.90 & 0.003 & 0.05 \\
SHRM area & 4.92 & 0.720 & 0.002 \\
SRF fluid area & 4.40 & 0.43 & 0.007 \\
\hline
\end{tabular}

One-year BCVA $=-1.098+0.327 \times$ baseline logMAR BCVA $+0.003145 \times$ central maximum thickness $+0.720 \times$ SHRM area

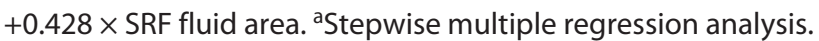

ed in comparison to an average choroidal thickness of $284.5 \pm 96.1 \mu \mathrm{m}$ in the AMD subgroup of the study by Singh et al. [19], which is relatively high for AMD patients. Choroidal thickness in AMD patients is usually thinner than average choroid with an average thickness below $200 \mu \mathrm{m}$ [20]. Thus, it is possible that part of AMD patients in the study by Singh et al. [19] had CNV secondary to PCV or CSR or peripapillary pachychoroid syndrome. In a small case series, Hamoudi et al. [21] examined 12 patients with PPCNV, 3 secondary to AMD and 9 idiopathic that were treated with a mean of 6.2 ranibizumab injections, and they found a BCVA improvement of 2 Snellen lines after treatment. In addition, 2 very small studies published an average of 3.5-4 anti-VEGF injections for 1 year [22, 23]. In contrast, Lin et al. [24] found mean BCVA at baseline in all eyes was $0.34 \pm 0.32 \log$ MAR (Snellen 20/44) that was maintained with a slight decrease to $0.60 \pm 0.51 \log$ MAR (Snellen 20/80) at the final visit $(p=0.159)$. Overall, according to this study and previous published studies, patients with PPCNV secondary to AMD may maintain or improve baseline BCVA after anti-VEGF injections $[8,19,21-24]$.

In this study, we examined CNV located on the temporal side of the optic disc and found that it was located in the superior half in $73.3 \%$ of the eyes. Similar to our findings, Singh et al. [19] found that $67.5 \%$ of the PPCNV was located in the superior temporal half of the optic disc. A possible explanation for this finding might be the impact of the superior half of the macula on the inferior visual field which is more noticeable for the patient than the superior visual field which is represented by the inferior half of the macula [25], thus there is a possible bias toward $\mathrm{CNV}$ lesions being symptomatic and therefore identified in the superior half. 
In this study, we found that eyes with greater CRT, min CRT, and max CRT had a significantly smaller improvement in BCVA at 1 year. In addition, eyes with a greater PED height, SHRM, PED, and SRF areas also had a significantly worse improvement in BCVA at 1 year. In multiple regression analysis, Singh et al. [19] found that age and baseline BCVA were significant predictors of change in BCVA through 12 months in PPCNV patients, but they did not examine OCT prognostic factors. We could not identify a previous PPCNV-related study that assessed OCT prognostic factors. However, previous studies that assessed subfoveal CNV secondary to AMD found morphological features on OCT, including IRF, SRF, and PED, to be significantly associated with baseline visual acuity and BCVA change. The VIEW study post hoc analysis found that IRF at baseline was the most important feature associated with both poor baseline visual acuity and lower visual improvement, while SRF at baseline was associated with better BCVA gain [26]. Simader et al. [11] reported as part of a subanalysis of the EXCITE study that PED predicted a poor visual outcome if combined with IRF or SRF. In a machine learning study, Schmidt-Erfurth et al. [27] found that the most relevant biomarker for BCVA was the horizontal extension of IRF in the foveal region, whereas SRF and PED ranked low. Post hoc analysis of the MARINA study found that larger CNV lesion size at baseline was negatively associated with visual acuity [28]. Similar to our findings, previous nAMD studies, including the CATT study, subanalysis found that greater foveal thickness at baseline predicted a lower overall BCVA at 12 months [29]. Most of these findings are similar to our current study findings and it can be deduced that OCT prognostic factors for PPCNV AMD related are similar to subfoveal CNV AMD related.

In this study, presence of ERM, hyper-reflective dots, and VMA were not found to be prognostic factors in terms of BCVA improvement. Similar to our findings, subanalysis of the EXCITE and MONT BLANC studies found that change in BCVA was not significantly different between patients PVD or VMA in either treatment arm $[30,31]$. However, in contrast to the current study, we previously reported HF to be a prognostic factor in subfoveal CNV secondary to AMD [10].

This study has several limitations, including its retrospective nature and relatively small study population. In addition, the location of SRF, IRF, and PED which may influence on visual acuity was not assessed.

It is important to note that we excluded patients with PPCNV secondary to other pathologies but AMD. In future, larger prospective studies may consider comparing the prognostic value of OCT findings in bevacizumab versus ranibizumab or aflibercept treatment. To the best of our knowledge, this is the first study reporting OCT prognostic factors in AMD-associated PPCNV treated with anti-VEGF.

\section{Conclusions}

Our data suggest that AMD-related PPCNV may have similar features to subfoveal CNV secondary to AMD in terms of OCT prognostic factors. Eyes with greater foveal thickness, PED size, SHRM, and SRF areas have worse final BCVA prognosis.

\section{Statement of Ethics}

All data for this were collected and analyzed in accordance with the policies and procedures of the Institutional Review Board (IRB) of the Meir Medical Center and the tenets set forth in the Declaration of Helsinki. Informed consent was not needed for this study.

\section{Conflict of Interest Statement}

No conflicting relationship exists for any author. The authors have no conflicts of interest to declare.

\section{Funding Sources}

The authors have no funding sources to declare.

\section{Author Contributions}

Gilad Rabina and Ori Segal designed the work; contributed to the acquisition, analysis, and interpretation of data for the work; drafted the manuscript; and revised it critically for important intellectual content. Anfisa Ayalon contributed to the acquisition and analysis of data for the work and drafted the manuscript. Michael Mimouni contributed to the acquisition, analysis, and interpretation of data for the work and drafted the manuscript. Nir Stanescu, Elad Moisseiev, Arie Y. Nemet, and Noa Geffen contributed to the analysis and interpretation of data for the work, drafted the manuscript, and revised it critically for important intellectual content. All authors approved the final version to be published.

\section{Data Availability Statement}

All data generated or analyzed during this study are included in this article. Further inquiries can be directed to the corresponding author.
Rabina/Ayalon/Mimouni/Stanescu/ Moisseiev/Nemet/Geffen/Segal 


\section{References}

1 Wilde C, Poostchi A, Mehta RL, Hillman JG, MacNab HK, Messina M, et al. Prevalence of peripapillary choroidal neovascular membranes (PPCNV) in an elderly UK population: the Bridlington eye assessment project (BEAP): a cross-sectional study (2002-2006). Eye. 2019 Mar;33(3):451-8.

2 Lopez PF, Green WR. Peripapillary subretinal neovascularization. A review. Retina. 1992;12(2):147-71.

3 Ruben S, Palmer H, Marsh RJ. The visual outcome of peripapillary choroidal neovascular membranes. Acta Ophthalmol. 1994 Feb;72(1):118-21.

4 Jutley G, Jutley G, Tah V, Lindfield D, Menon G. Treating peripapillary choroidal neovascular membranes: a review of the evidence. Eye. 2011 Jun;25(6):675-81.

5 Turcotte P, Maguire MG, Fine SL. Visual results after laser treatment for peripapillary choroidal neovascular membranes. Retina. 1991;11(3):295-300.

6 Almony A, Thomas MA, Atebara $\mathrm{NH}$, Holekamp NM, Del Priore LV. Long-term follow-up of surgical removal of extensive peripapillary choroidal neovascularization in presumed ocular histoplasmosis syndrome. Ophthalmology. 2008 Mar;115(3): 540-e5.

7 Bernstein PS, Horn RS. Verteporfin photodynamic therapy involving the optic nerve for peripapillary choroidal neovascularization. Retina. 2008 Jan;28(1):81-4.

8 Yun C, Oh J, Choi KE, Hwang SY, Kim SW, Huh K. Peripapillary choroidal thickness after intravitreal ranibizumab injections in eyes with neovascular age-related macular degeneration. BMC Ophthalmol. 2016 Dec; 16(1):25

9 Manjunath V, Goren J, Fujimoto JG, Duker JS. Analysis of choroidal thickness in agerelated macular degeneration using spectral-domain optical coherence tomography. Am J Ophthalmol. 2011 Oct;152(4): 663-8.

10 Segal O, Barayev E, Nemet AY, Geffen N, Vainer I, Mimouni M. Prognostic value of hyperreflective foci in neovascular age-related mascular degeneration treated with bevacizumab. Retina. 2016 Nov;36(11): 2175-82.

11 Simader C, Ritter M, Bolz M, Deák GG, Mayr-Sponer U, Golbaz I, et al. Morphologic parameters relevant for visual outcome during anti-angiogenic therapy of neovascular age-related macular degeneration. Ophthalmology. 2014;121(6):1237-45.
12 Lai TT, Hsieh YT, Yang CM, Ho TC, Yang $\mathrm{CH}$. Biomarkers of optical coherence tomography in evaluating the treatment outcomes of neovascular age-related macular degeneration: a real-world study. Sci Rep. 2019 Dec;9(1):529.

13 Talks J, Koshy Z, Chatzinikolas K. Use of optical coherence tomography, fluorescein angiography and indocyanine green angiography in a screening clinic for wet agerelated macular degeneration. Br J Ophthalmol. 2007 May;91(5):600-1.

14 Hosseini H, Rabina G, Pettenkofer M, Au A, Chehaibou I, Heilweil G, et al. Clinical characteristics and visual outcomes of nonresolving subretinal fluid in neovascular AMD despite continuous monthly antiVEGF injections: a long-term follow-up. Graefes Arch Clin Exp Ophthalmol. 2021 May;259(5):1153-60.

15 Regatieri CV, Branchini L, Duker JS. The role of spectral-domain OCT in the diagnosis and management of neovascular agerelated macular degeneration. Ophthalmic Surg Lasers Imaging. 2011;42(Suppl 0):S56.

16 Mrejen S, Sarraf D, Mukkamala SK, Freund KB. Multimodal imaging of pigment epithelial detachment: a guide to evaluation. Retina. 2013 Oct;33(9):1735-62.

17 Dansingani KK, Tan ACS, Gilani F, Phasukkijwatana N, Novais E, Querques L, et al. Subretinal hyperreflective material imaged with optical coherence tomography angiography. Am J Ophthalmol. 2016 Sep; 169:235-48.

18 Keane PA, Liakopoulos S, Chang KT, Wang M, Dustin L, Walsh AC, et al. Relationship between optical coherence tomography retinal parameters and visual acuity in neovascular age-related macular degeneration. Ophthalmology. 2008 Dec;115(12):220614.

19 Singh SR, Fung AT, Fraser-Bell S, Lupidi M, Mohan S, Gabrielle PH, et al. One-year outcomes of anti-vascular endothelial growth factor therapy in peripapillary choroidal neovascularisation. Br J Ophthalmol. 2020 May;104(5):678-83.

20 Chung SE, Kang SW, Lee JH, Kim YT. Choroidal thickness in polypoidal choroidal vasculopathy and exudative age-related macular degeneration. Ophthalmology. 2011 May;118(5):840-5.

21 Hamoudi H, Sørensen TL. Effect of intravitreal ranibizumab in the treatment of peripapillary choroidal neovascularisation. J Ophthalmol. 2011;2011:1-4.

22 Adrean SD, Grant S, Chaili S. Bevacizumab (avastin) and thermal laser combination therapy for peripapillary choroidal neovascular membranes. J Ophthalmol. 2017; 2017:4802690.
23 Figueroa MS, Noval S, Contreras I. Treatment of peripapillary choroidal neovascular membranes with intravitreal bevacizumab. Br J Ophthalmol. 2008 Sep;92(9): 1244-7.

24 Lin T, Dans K, Meshi A, Muftuoglu IK, Amador-Patarroyo MJ, Chen KC, et al. Age-related macular degeneration-associated peripapillary choroidal neovascularization in the era of anti-vascular endothelial growth factor therapy. Retina. 2019 Oct;39(10): 1936-44.

25 Black AA, Wood JM, Lovie-Kitchin JE. Inferior visual field reductions are associated with poorer functional status among older adults with glaucoma. Ophthalmic Physiol Opt. 2011 May;31(3):283-91.

26 Waldstein SM, Simader C, Staurenghi G, Chong NV, Mitchell P, Jaffe GJ, et al. Morphology and visual acuity in aflibercept and ranibizumab therapy for neovascular agerelated macular degeneration in the VIEW trials. Ophthalmology. 2016 Jul;123(7): 1521-9.

27 Schmidt-Erfurth U, Bogunovic H, Sadeghipour A, Schlegl T, Langs G, Gerendas BS, et al. Machine learning to analyze the prognostic value of current imaging biomarkers in neovascular age-related macular degeneration. Ophthalmol Retin. 2018;2(1):2430.

28 Boyer DS, Antoszyk AN, Awh CC, Bhisitkul RB, Shapiro H, Acharya NR. Subgroup analysis of the MARINA Study of ranibizumab in neovascular age-related macular degeneration. Ophthalmology. 2007 Feb; 114(2):246-52.

29 Singh RP, Fu EX, Smith SD, Williams DR, Kaiser PK. Predictive factors of visual and anatomical outcome after intravitreal bevacizumab treatment of neovascular age-related macular degeneration: an optical coherence tomography study. Br J Ophthalmol. 2009 Oct;93(10):1353-8.

30 Waldstein SM, Ritter M, Simader C, MayrSponer U, Kundi M, Schmidt-Erfurth U. Impact of vitreomacular adhesion on ranibizumab mono- and combination therapy for neovascular age-related macular degeneration. Am J Ophthalmol. 2014;158(2): 328

31 Mayr-Sponer U, Waldstein SM, Kundi M, Ritter M, Golbaz I, Heiling U, et al. Influence of the vitreomacular interface on outcomes of ranibizumab therapy in neovascular age-related macular degeneration. Ophthalmology. 2013 Dec;120(12):26209. 